\title{
Uma Integração entre Ferramentas de Gerência de Projetos Utilizando XML
}

\author{
Edson Leandro de Araújo Silva², Adailton Magalhães Lima ${ }^{1,2}$, Ernani de Oliveira \\ Sales $^{1}$, Anderson Jorge Serra da Costa ${ }^{2}$
}

${ }^{1}$ Laboratório de Engenharia de Software - Universidade Federal do Pará (UFPA)

Belém - PA- Brasil

${ }^{2}$ Faculdade de Sistemas de Informação - Universidade Federal do Pará (UFPA)

Castanhal - PA - Brasil

edsonlead@gmail.com, \{adailton,ernani\}@ufpa.br, anderson@webapsee.com

\begin{abstract}
This paper describes a research to develop an integration between tools that support the management of software development projects. One of the main motivations for this work is that there is a regular need of software companies to use a combination of complementary tools to help them control and manage their software projects. However, the heterogeneity of project management tools creates a hard way to integrate their information. Therefore, this paper presents a solution to integrating project management tools using XML technology. We present the result of the integration of two tools using the proposed solution as a way to demonstrate the feasibility of the project.
\end{abstract}

Resumo. Este artigo descreve um trabalho de pesquisa, cujo o tema está relacionado às ferramentas utilizadas para auxiliar a gerência de projetos de desenvolvimento de software. É frequente a necessidade destas organizações utilizarem uma combinação de ferramentas complementares para auxiliar o controle e administração de seus projetos. Porém, a heterogeneidade das ferramentas de gerenciamento de projetos é um fator que complica as organizações de integrar suas informações. Portanto, este artigo apresenta uma solução para o problema de integração entre sistemas gestoras de projetos utilizando a tecnologia XML. E apresentado o resultado da integração de duas ferramentas utilizando a solução proposta como forma de demonstrar a viabilidade do projeto.

\section{Introdução}

No contexto de desenvolvimento de software existem diversos processos que apóiam o gerenciamento das atividades de Engenharia de Software e no desenvolvimento de sistemas, que são as ferramentas de gerenciamento de projetos utilizadas na administração, controle e gerência dos projetos. Porém, a heterogeneidade dessas ferramentas de gerência de projetos é um fator que complica a integração entre elas.

Para [Nascimento 2001] integrar sistemas significa fazer com que estes possam trocar informações de maneira a interoperável e visando atingir um objetivo comum. A integração, o compartilhamento de dados, entre ferramentas de gerência de projetos pode ser necessário para as organizações que utilizam diferentes softwares de maneira 
combinada por apenas uma não possuir todas as funcionalidades que a organização necessita em seus projetos ou para o uso de outros setores da organização, como o RH para gerenciar as horas trabalhadas dos funcionários, por exemplo.

Quando trata-se de integração entre ferramentas a tecnologia XML é apontada como uma possível solução para o caso. Segundo [Kay 2002], o XML é uma linguagem simples e padronizada pra a troca de informações entre programas de computador. Geralmente, as ferramentas possuem funcionalidade de exportar dados do projeto em documentos XML e de importá-los nas mesmas, esses documentos XML armazenam os dados em uma estrutura XML padronizada pelos seus fornecedores e são denominados de modelos de dados. Cada ferramenta possui um modelo de dados próprio e diferente de seus concorrentes.

$\mathrm{O}$ fato de cada ferramenta de gerenciamento de projetos possuir o seu próprio modelo de dados aumenta o nível de dificuldade de integrá-las, pois para que a integração ocorra os esquemas XML devem ser manipulados para averiguar a compatibilidade entre as suas marcações XML. Quantos mais marcações compatíveis mais dados podem ser compartilhados e menos perda de dados terá durante a integração entre as ferramentas. Uma solução para isso é os fornecedores de softwares utilizarem um único modelo de dados padrão em suas ferramentas.

E dentro desse contexto de padronização de modelo de dados para a integração entre ferramentas de apoio às atividades envolvidas no processo de Engenharia de Software é que o Modelo MIIPS (Modelo Interoperável para Compartilhamento de Informações em Gerência de Projetos de Software) foi desenvolvido em um projeto do LABES/UFPA (Laboratório de Engenharia de Software da Universidade Federal do Pará), denominado de "Modelo Interoperável para Compartilhamento de Informações em Gerência de Projetos de Software". Um dos objetivos desse artefato, segundo [Costa 2009] é que o mesmo seja um padrão comum que possa ser utilizado por diversos fornecedores de software para a gerência de projetos como forma de minimizar a dificuldade de integração existente entre as diferentes ferramentas disponíveis no mercado. Na seção 3.2 deste artigo serão descritos mais detalhes sobre este modelo.

No projeto citado, o MIIPS foi desenvolvido e proposto o compartilhamento de dados entre a ferramenta MS Project [López 2008] e o modelo MIIPS (este compartilhamento de dados se dá através de transformações de documentos, que será descrito ao decorrer do texto). A ferramenta WebAPSEE [Carvalho 2011] também foi utilizada para se ter um ciclo total de compartilhamento de dados entre duas ferramentas de gerenciamento de projetos utilizando o modelo MIIPS.

É comum dentro de organizações existir ambientes heterogêneos, se tratando de ferramentas e plataformas de diferentes fornecedores por necessidade dos projetos. $\mathrm{O}$ gestor pode precisar em algum momento de uma solução de integração entre ferramentas que diminua o trabalho de gerenciar vários documentos de diferentes ferramentas. Enquanto existirem várias ferramentas gestoras de projetos existe a possibilidade de compartilhar dados entre elas mediante o uso do modelo MIIPS. Tornando-o um modelo padrão de interoperabilidade de software, de forma análoga, tornar o MIIPS para a integração de ferramentas de gerenciamento de projetos o que o XMI [Yang 2005] é para a UML.

Os objetivos deste trabalho são apresentar o compartilhamento de dados entre as ferramentas MS Project e WebAPSEE, e também o modelo de integração entre ferramentas de gerência de projetos de software através do MIIPS. Dessa forma, o 
objetivo geral é desenvolver uma solução de integração apresentando o MIIPS como Modelo Padrão de Interoperabilidade de Software, para que mais ferramentas possa utilizá-lo como solução para a dificuldade de integrar ferramentas de gerenciamento de projetos. Além de diminuir a dificuldade na integração de ferramentas, o MIIPS objetiva diminuir os esforços de migração de ferramentas dentro do ambiente organizacional, onde já se possui uma cultura organizacional instalada. Evitando, também, a substituição de ferramentas e integrar funcionalidades de ferramentas, enriquecendo todo o conjunto para o processo de desenvolvimento de software.

Como já citado, para que haja o compartilhamento de dados faz-se necessário a transformação de modelos de dados XML. E para isso a importância do uso da Tecnologia XML/XSLT para a transformação desses documentos. Para tal solução foi desenvolvido uma ferramenta intermediária que realiza a comunicação entre as ferramentas e o MIIPS.

\section{Metodologia}

Foram realizadas atividades relacionadas atividades de projeto e prototipagem dos parsers de integração para cumprir os objetivos do trabalho de pesquisa. As atividades seguidas foram: Estudo sobre o modelo XML do MS Project; Estudo sobre o modelo MIIPS; Estudo sobre o modelo XML do WebAPSEE; Projeto Arquitetural da solução de integração proposta; Implementação do parser de integração; Realização de testes de software na solução desenvolvida; Estudo de caso com dados de um projeto real como fonte de dados para testar as transformações entre os modelos.

\section{Solução Proposta}

A solução proposta para a integração entre as ferramentas MS Project e WebAPSEE foi o uso de documentos no formato padrão XML dessas ferramentas para a transformação entre eles em conjunto com o modelo MIIPS.

Para que aconteça as conversões/transformações dos documentos XML foi proposto o desenvolvimento de templates XSL (item 3.1) em um total de quatro templates para o compartilhamento de dados entre as ferramentas e o MIIPS. Na Tabela 1 estão listados esses quatros templates com seus respectivos documentos que serão transformados (origem) e os resultados de cada transformação (destino).

Tabela 1. Templates XSL da Solução Proposta

\begin{tabular}{|l|c|c|}
\hline \multicolumn{1}{|c|}{ Template XSL } & Origem & Destino \\
\hline $\begin{array}{l}\text { Template XSL de transformação MS Project } \\
\text { MIIPS }\end{array}$ & MS Project & MIIPS \\
\hline $\begin{array}{l}\text { Template XSL de transformação WebAPSEE } \\
\text { MIIPS }\end{array}$ & WebAPSEE & MIIPS \\
\hline $\begin{array}{l}\text { Template XSL de transformação MIIPS MS } \\
\text { Project }\end{array}$ & MIIPS & MS Project \\
\hline $\begin{array}{l}\text { Template XSL de transformação MIIPS } \\
\text { WebAPSEE }\end{array}$ & MIIPS & WebAPSEE \\
\hline
\end{tabular}

O Diagrama de Casos de Uso a seguir (Figura 1), ilustra as funcionalidades propostas para o projeto em questão. Onde possivelmente pode ser observado que o ator Usuário possui opções relacionadas as transformações entre os modelos das ferramentas 
e o MIIPS para que ocorra o compartilhamento de dados.

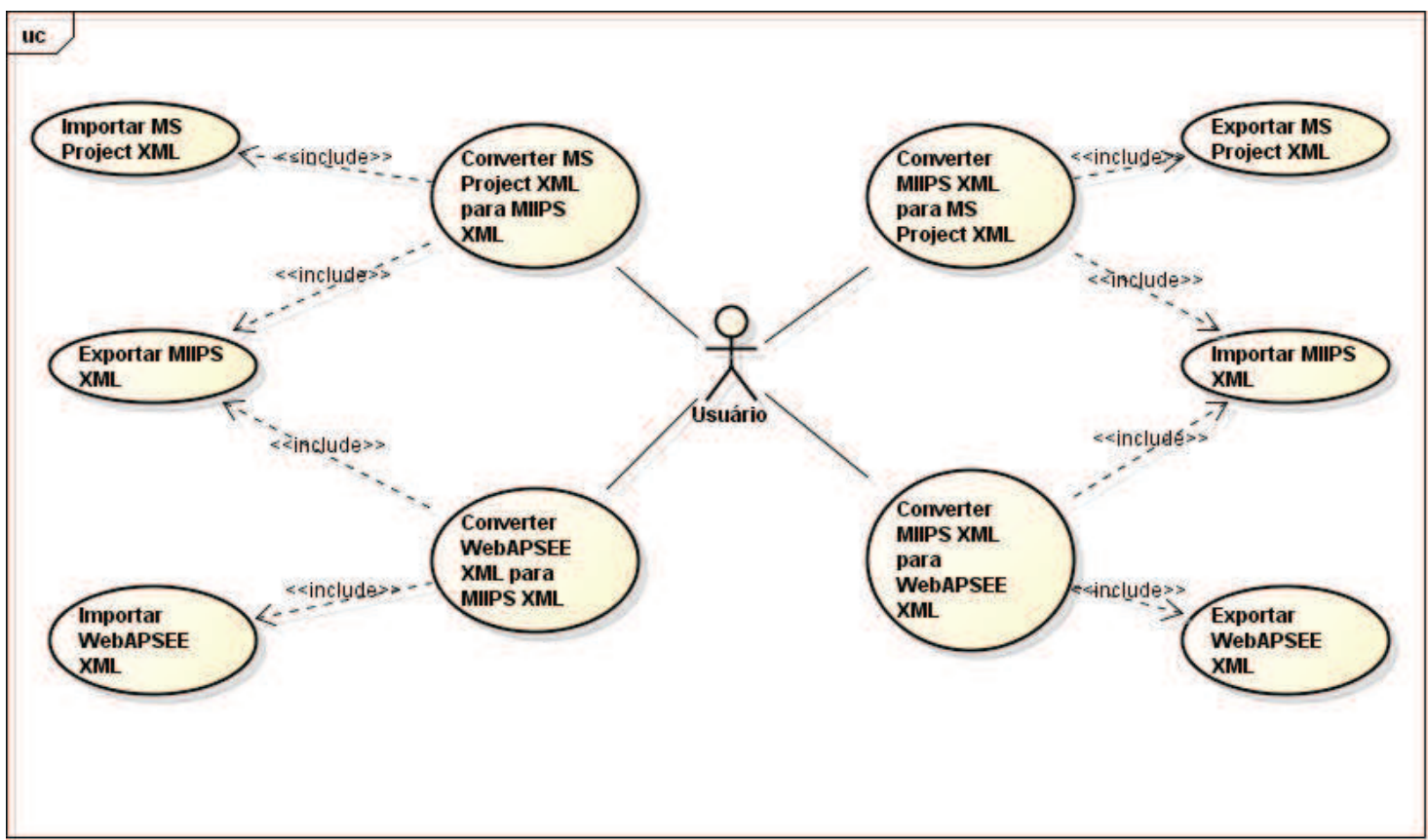

Figura 1. Diagrama de Casos de Uso relativo ao parser de transformação

\subsection{Tecnologia XML/XSLT}

O XML (eXtensible Markup Language) é uma linguagem padrão de marcação recomendada pela W3C (World Wide Web Consortium) para estruturar dados e informações na Web, porém, também é utilizada em ferramentas Desktops. A sua estrutura de marcações é em formato de árvore, ou seja, possui nós-filhos de um nó-pai, e a sua estrutura é flexível a ponto do próprio desenvolvedor poder criar os próprios marcadores para um documento, daí a possibilidade de cada ferramenta possuir um modelo de dados próprio.

Essa flexibilidade do XML ajuda na criação de documentos inteligentes, que ajudam a separar o conteúdo da apresentação dos documentos, fazendo com que o conteúdo possa ser apresentado de várias formas através do XSLT (eXtensible Stylesheet Language Transformation). Segundo [Kay 2002], o XSLT "é uma linguagem que [...] foi projetada principalmente para transformar um documento XML em outro [...]" e ainda reforça que o "XSLT é uma linguagem para transformar a estrutura de um documento XML". Ou seja, a transformação com o uso do XSLT pode ser utilizada para transformar um documento XML em um documento com formato diferente, HTML por exemplo, ou em outro XML com sua estrutura diferente.

O XSLT trabalha em conjunto com o XSL (eXtensible Stylesheet Language Transformation), que nada mais é do que folha de estilo. O XSL que dita como o documento XML será apresentado, basicamente, o XSL é para o XML o que o CSS é para o HTML. O XSLT implementa o XSL no documento XML e o transforma em outro formato ou estrutura. Para este trabalho, a transformação entre documentos XML apenas afetará a estrutura do XML de cada um documento, para que possa ser suportado pelas ferramentas que suportam apenas documentos com as respectivas estruturas de seus modelos de dados. 


\subsection{Modelo MIIPS}

O modelo MIIPS é um modelo de dados desenvolvido em um projeto do LABES/UFPA em 2009, patrocinado pela Microsoft e derivado do WebAPSEE e do SPEM (Definition of an Executable SPEM 2.0). O modelo objetiva ser um padrão para o compartilhamento de dados entre ferramentas de gerência de projetos, de forma análoga, ser o que o XMI é para o UML. As suas propostas, segundo [Costa 2009], são as seguintes: Compartilhamento de dados entre ferramentas; Agregar informações de contextualização do projeto; Maior percepção do desenvolvedor em relação a sua participação no projeto como um todo; A mesma informação em qualquer lugar; Disponibilização das informações na Web (2.0) e SmartPhones.

A Figura 2 ilustra um pedaço do Diagrama de Classes do modelo MIIPS. O MIIPS procura ser mais genérico possível em relação aos outros modelos de dados das ferramentas disponibilizadas no mercado. Ou seja, significa que este é um modelo que abrange diferentes ferramentas, sendo possível armazenar dados de: projetos, tarefas, atividades, artefatos, recursos humanos e materiais, paradigmas, processos e outros.

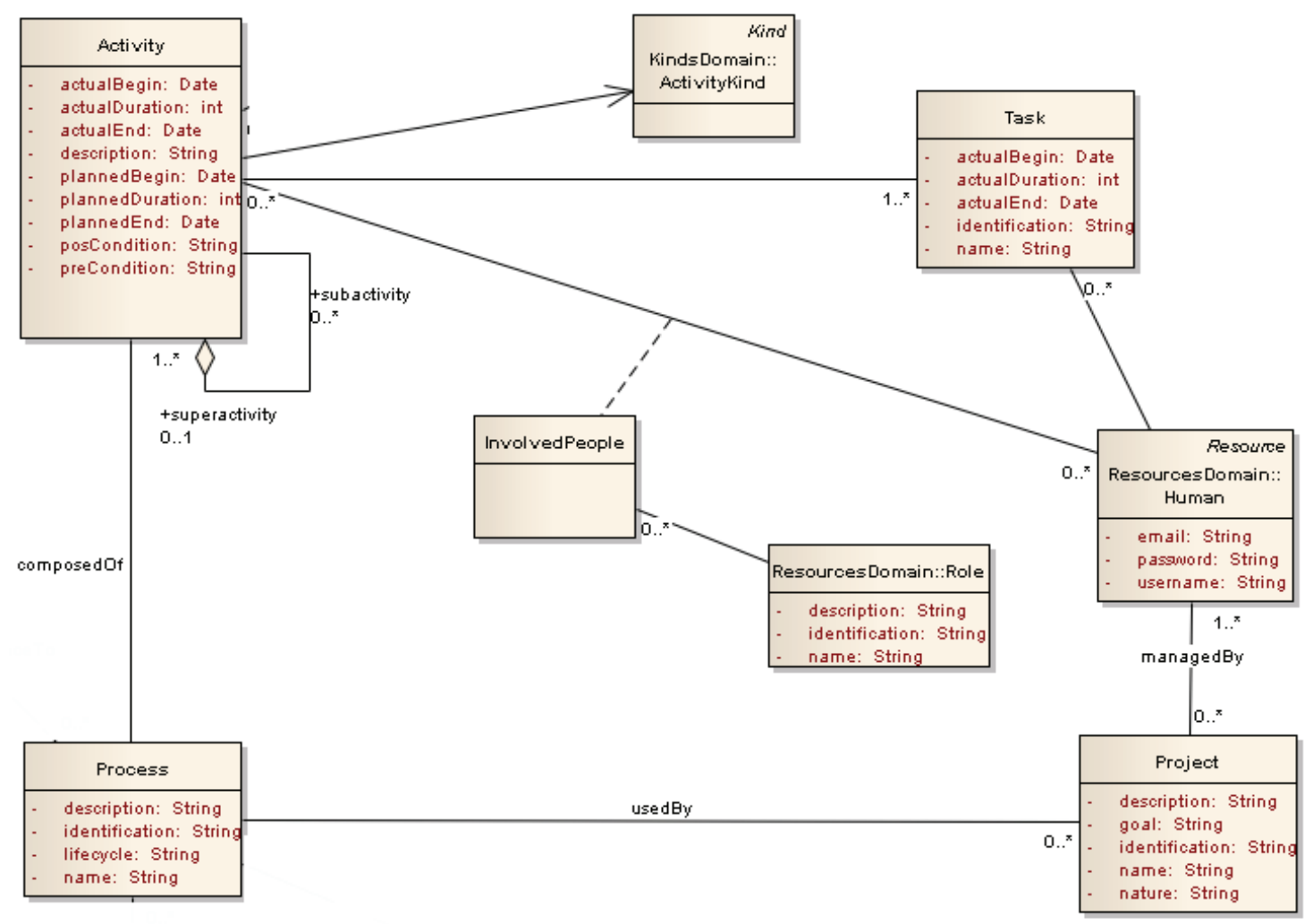

Figura 2. Parte do Diagrama de Classes do Modelo MIIPS

As entidades do modelo MIIPS armazenam dados de si, a destacar as principais entidades utilizadas pelo MIIPS e pertencentes as ferramentas gestoras de projetos que estão no mercado, são elas: Projeto, Atividade, Tarefa, Recursos Materiais e Humanos. O Projeto é o empreendimento que deve ser, controlado, administrado e planejado de forma cuidadosa e dividida em Atividades (e essas Atividades podem ser divididas em Tarefas).Para chegar ao objetivo do Projeto deve-se possuir uma equipe (Recursos Humanos) equipada com ferramentas (Recursos Materiais) necessárias para resolver as 
Tarefas de cada Atividade.

O MIIPS possuir estrutura similar as outras ferramentas e se tratadas da forma apresentada neste trabalho torna-se possível gerar um documento XML MIIPS para várias ferramentas de gestão de projetos e reunir funcionalidades das ferramentas em apenas um documento XML. Na Figura 3 é ilustrado um trecho do schema XML do MIIPS, onde pode ser observado um trecho do schema do MIIPS referente à classe Task (Tarefas) da Figura 2.

\section{exs :element name="Task" type="Task"/> \\ exs: complexType nane="Task"> \\ exs:sequences \\ exs:element name="identification" type="xs: string" minoccurs="l" maxOccurs="l"/> \\ exs:element name="name" type="xsistring" minoccurs="1" naxOccurs="l"/> \\ exs:element name="actualBegin" type="xs: date" ninoccurs="1" maxOccurs="1" $/>$ \\ <xs:element name="actualOuration" type="xs: int" "minoccurs="1" maxOccurs="1" $1>$ \\ <xs:element name="actualEnd" type="xs:date" minoccurs="l" maxOccurs $=" 1 " />$ \\ <xs:element name="Activity" type="Activity" minoccurs="l" maxOccurs="l"/> \\ exs:element name="Human" type="Human" minOccurs="l" maxOccurs="l" \\ $</ x$ s: sequences \\ $\langle/ x$ s: complexType $\rangle$}

Figura 3. Trecho do schema XML do modelo MIIPS

O modelo MIIPS busca ser um padrão de interoperabilidade de software dentro do contexto de Processo de Desenvolvimento de Software, o que facilitará a integração entre as ferramentas gestoras de projetos.

\subsection{Ferramentas Envolvidas}

Para apresentar o modelo MIIPS como modelo padrão de interoperabilidade de software, o mesmo é utilizado como modelo intermediário entre ferramentas. As ferramentas pioneiras dessa solução de integração serão apresentadas a seguir.

\subsubsection{MS Project Professional 2010}

O MS Project é uma ferramenta de gerência de projetos desenvolvido pela Microsoft. Em [López 2008], é reforçado a organização que a ferramenta possui em relação as tarefas e aos custos tanto da mão de obra quanto do material. Sendo as tarefas e prazos o seu foco principal.

Sua primeira versão foi lançada em 1984 para ambiente DOS para facilitar o controle de tarefas e projetos de uma forma prática e rápida. Em 1990, recebeu a primeira versão para o sistema operacional Windows. Em 1995 foi apresentado como Microsoft Office Project apesar de nunca ter sido incluído no pacote de Suítes de Escritórios da empresa. Atualmente está na versão 2013, embora tenha sido escolhida a versão 2010 para este projeto devido a razões internas ao projeto.

A escolha da utilização desta ferramenta no trabalho de pesquisa, dá-se pelo motivo de a Microsoft ter patrocinado o projeto MIIPS e devido ao MS Project ser a 
ferramenta de gerência de projetos mais utilizada no Brasil [PMI 2010]. Segundo a pesquisa realizada em 2010 pelo PMI (Project Management Institute), com 460 organizações, para verificar o perfil do Gerenciamento de Projetos no Brasil, no quesito softwares para o apoio de gerenciamento de projetos mais utilizados, o destaque foi para o MS Project com $76.1 \%$ dentro de um total de $81 \%$ das organizações brasileiras que utilizam alguma ferramenta de gerência de projetos. Em quarto lugar na pesquisa, o MS Project Server aparece com 27.6\%.

\subsubsection{WebAPSEE}

Segundo [Carvalho 2011], o WebAPSEE foi desenvolvido pelo Laboratório de Engenharia de Software da UFPA (LABES/UFPA) para o apoio ao desenvolvimento de software centrado em processos. O ambiente WebAPSEE foi desenvolvido com uma arquitetura distribuída e com o uso de web services para prover portabilidade entre diferentes plataformas, linguagens e a comunicação com outras ferramentas. A figura a seguir (Figura 4) ilustra uma modelagem de processo na ferramenta WebAPSEE.

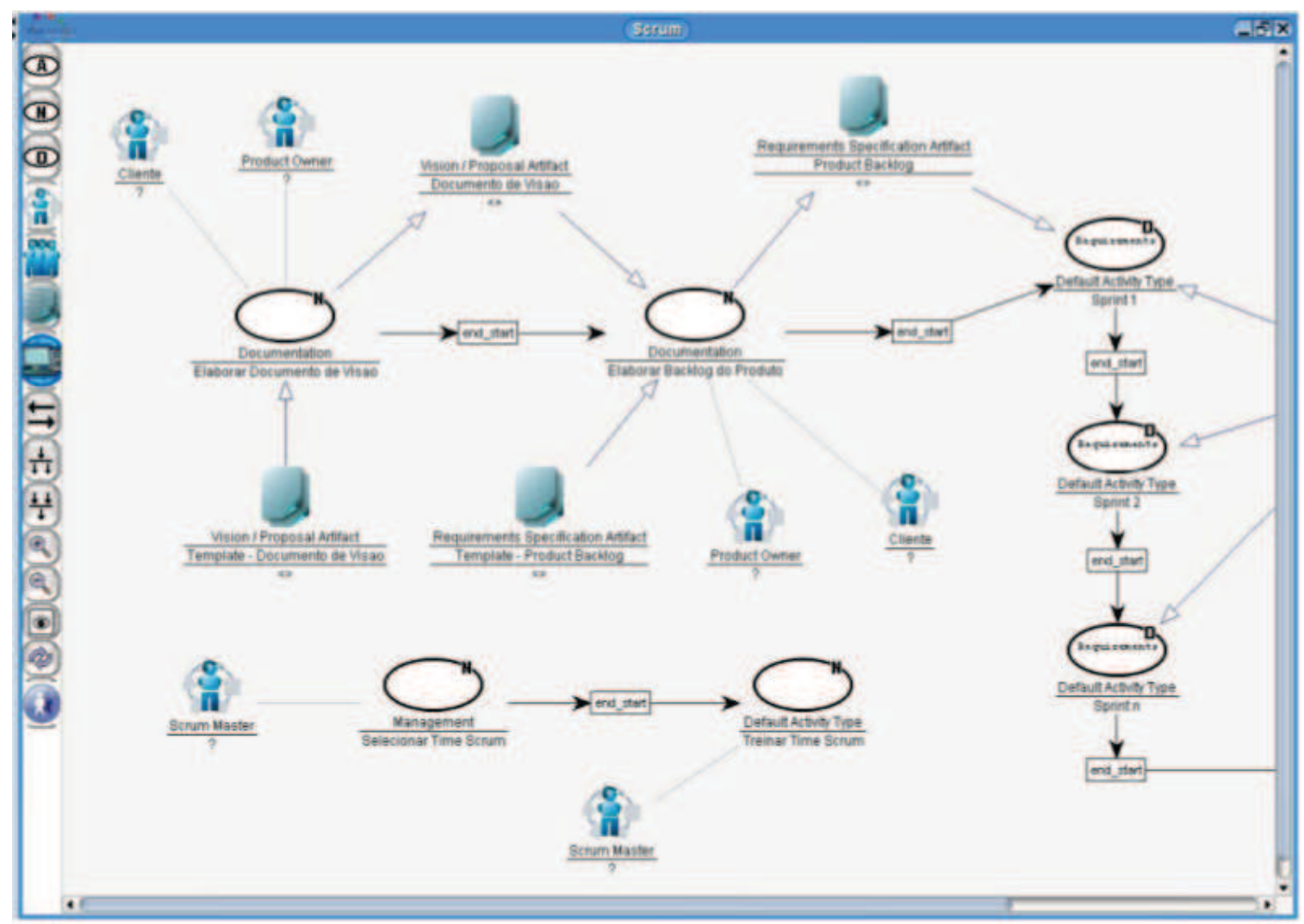

Figura 4. Processo aberto na ferramenta WebAPSEE

Permitindo assim que o WebAPSEE modele, execute e acompanhe os processos por meio de uma Linguagem Visual de Modelagem de Processos (WebAPSEE-PML), o que facilita a reutilização das boas práticas gerenciais por diferentes projetos.

O WebAPSEE foi inserido na trabalho de pesquisa para que houvesse um ciclo total de integração entre duas ferramentas de gerência de projetos. A escolha da ferramenta foi devido a mesma, assim como o MIIPS, ter sido desenvolvida no LABES/UFPA. 


\section{Resultados do Trabalho}

Neste trabalho foram desenvolvidos alguns artefatos como: especificação do modelo MIIPS, esquema XML do MIIPS, mapeamento conceitual do MS Project e MIIPS, lista de requisitos do caso de uso, projeto de arquitetura, mapeamento conceitual WebAPSEE e MIIPS, templates XSL e parser de Transformação, e por último, o protótipo funcional. $\mathrm{E}$ foi utilizado um modelo XML da ferramenta WebAPSEE contendo dados reais de um projeto, que inicialmente foi transformado manualmente para o modelo MIIPS (pois, ainda não se havia implementado o template XSL responsável por tal transformação). Com a transformação citada, obteve-se um modelo MIIPS com dados reais de um projeto, este foi transformado com o parser de transformação para o MS Project.

Foi verificado que a ferramenta WebAPSEE não possui suas atividades e tarefas de forma ordenada por data, como acontece no MS Project. Sem essa ordenação, quando transformar um documento de WebAPSEE para MIIPS e depois para MS Project, o resultado será um documento MS Project, este não realizará o cálculo automático de tempo de duração do projeto, pois, o MS Project se utiliza da ordenação das tarefas por data para realizar esse cálculo. Essa limitação foi tratada na transformação feita manualmente do modelo XML do WebAPSEE para o modelo XML do MIIPS. Não foi tratado o caso de existência de ativades paralelas em projetos.

Os quatros templates XSL que foram propostos (item 3) para a transformação dos documentos foram desenvolvidos, satisfazendo o Diagrama de Caso de Uso (Figura 1) e o Diagrama de Atividades (Figura 2).

A transformação do documento XML da ferramenta WebAPSEE para o XML MIIPS e depois para o XML MS Project foi devidamente realizada e retornou resultados satisfatórios ao importar o documento gerado para a ferramenta MS Project. Porém, a transformação de volta do XML MS Project para MIIPS e depois para o XML WebAPSEE não retornou o resultado esperado, pois a ferramenta WebAPSEE não suportou o documento XML WebAPSEE gerado.

\subsection{Templates de transformação entre os modelos do MS Project e MIIPS}

A implementação dos templates XSL responsáveis pelas tranformações entre os modelos MS Project e MIIPS foram desenvolvidos após os estudos entre os modelos envolvidos, levando em consideração o mapeamento conceitual dos modelos.

Foram desenvolvidos, neste trabalho, dois templates XSL responsáveis pelas transformações entre esses modelos: o template XSL de transformação MS Project em MIIPS e o template XSL de transformação MIIPS em MS Project. O primeiro é responsável por capturar os dados do modelo MS Project e adicioná-los à estrutura do modelo XML do MIIPS. E o último, faz o contrário, captura os dados do modelo MIIPS e adiciona-os à estrutura do modelo XML do MS Project. O desenvolvimento desses templates estão de acordo com a compatibilidade entre as marcações descritas no mapeamento conceitual.

Na Figura 5 está ilustrado um trecho do template responsável pela transformação de MIIPS para MS Project. As marcações XML que podem ser vista na Figura 5 fazem parte da estrutura XML do modelo de dados MS Project, enquanto o XSL faz a captura dos dados MIIPS compatíveis com cada marcação MS Project. 


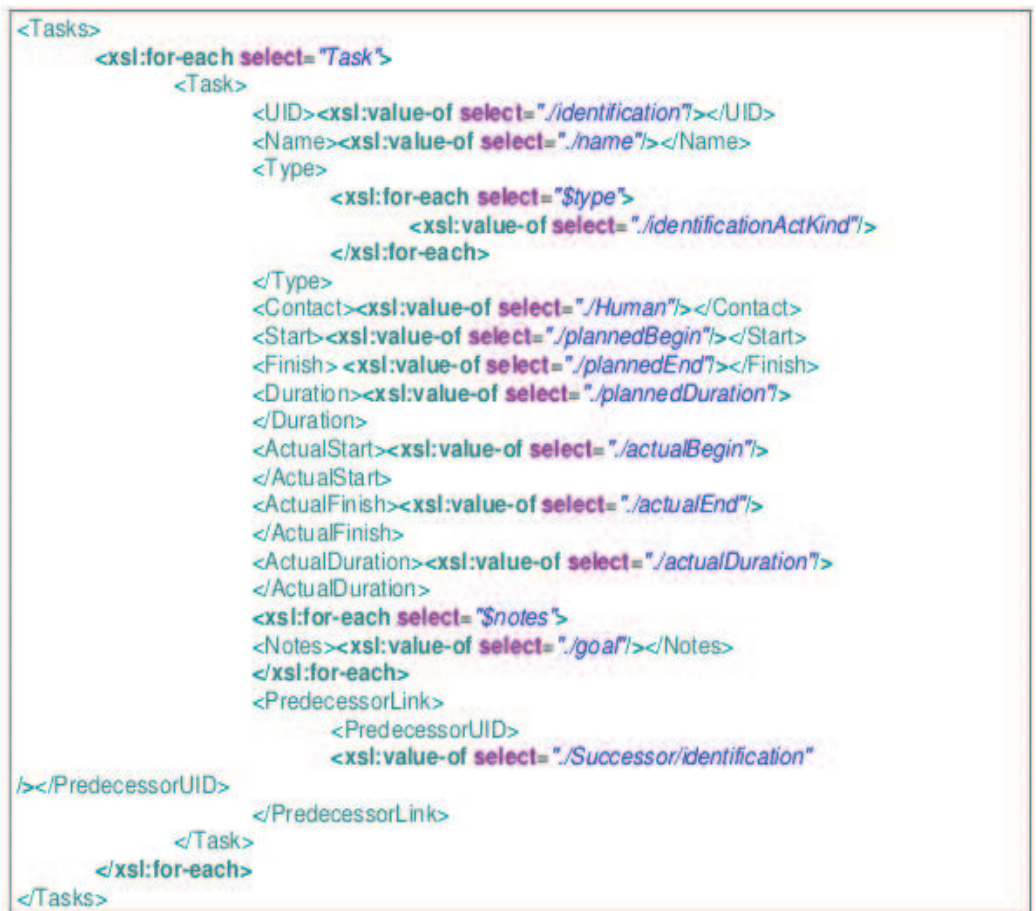

Figura 5. Trecho do template XSL da transformação MIIPS em MS Project

Com um documento de entrada MIIPS e com a execução do template ilustrado na Figura 5, foi obtido um documento MS Project como resultado da transformação. Na figura a seguir (Figura 6) tem-se o documento MS Project gerado, no trabalho, a partir de um documento MIIPS com dados reais de um projeto e importado para a ferramenta MS Project Professional 2010.

Para a Figura 6 foi escolhida o tipo de visualização Calendário, para ilustrar que o MS Project suportou o documento XML gerado pela transformação. Na Figura 6, pode-se visualizar as tarefas de acordo com o seu mês, semana e de forma personalizada pelo gestor. 


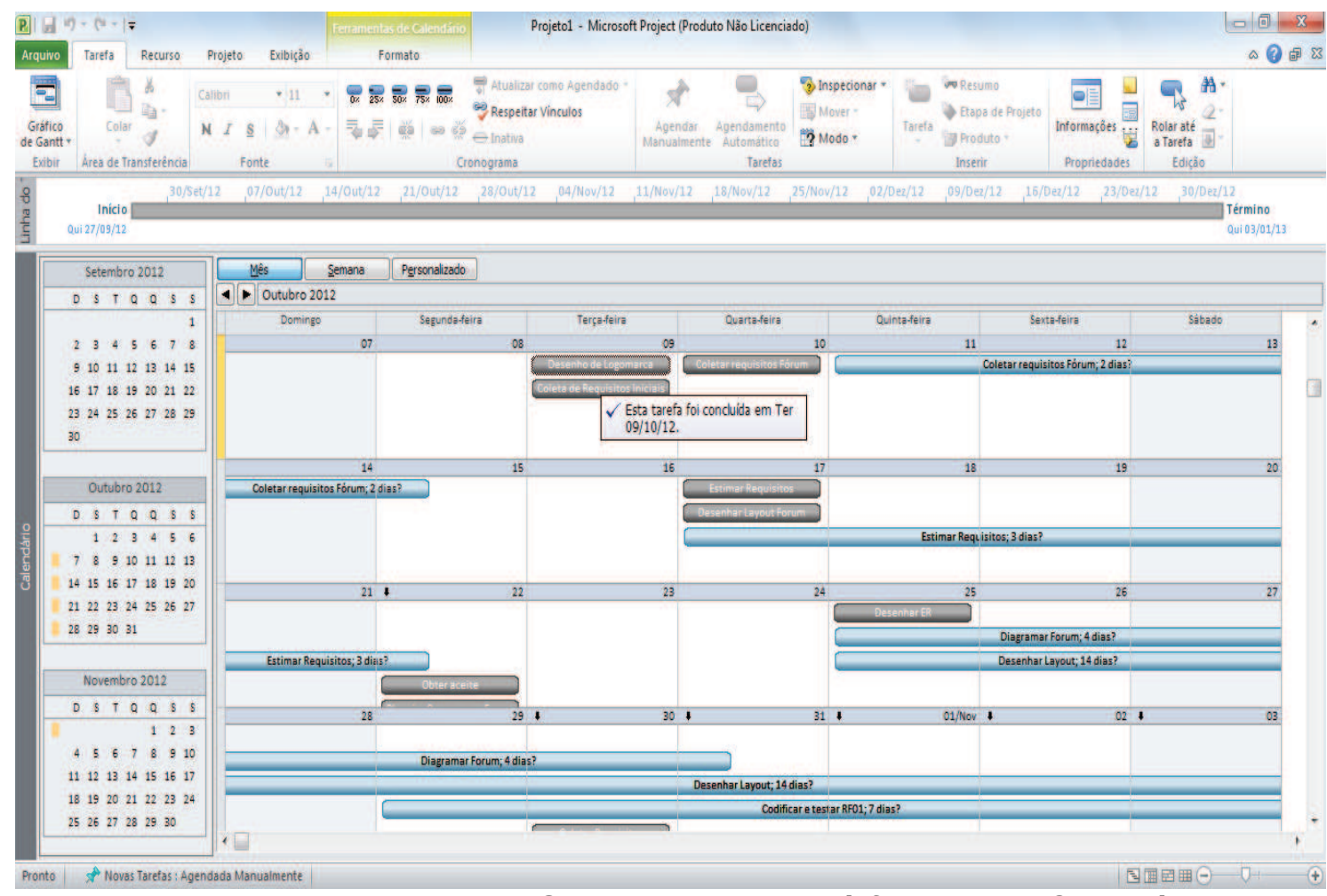

Figura 6. Documento XML MS Project importado à ferramenta (Calendário)

\subsection{Templates de transformação entre os modelos do WebAPSEE e MIIPS}

A implementação dos templates XSL responsáveis pelas transformações entre os modelos WebAPSEE e MIIPS foram desenvolvidos após os estudos entre esses modelos envolvidos, levando em consideração a transformação feita manualmente de WebAPSEE para MIIPS que auxiliou no desenvolvimento do mapeamento conceitual entre os modelos citados.

Assim como no item anterior (item 4.1), também foram desenvolvidos dois templates XSL responsáveis pela transformação entre os modelos WebAPSEE e MIIPS: o template XSL de transformação WebAPSEE em MIIPS e o template XSL de transformação MIIPS em WebAPSEE. O primeiro é responsável por capturar os dados do modelo WebAPSEE e adicioná-los à estrutura do modelo XML do MIIPS. E o último é responsável por capturar os dados do modelo MIIPS e adicioná-los à estrutura do modelo XML do WebAPSEE. Os templates foram desenvolvidos levando em consideração a compatibilidade entre as marcações que foram definidas no mapeamento conceitual entre os modelos envolvidos. 


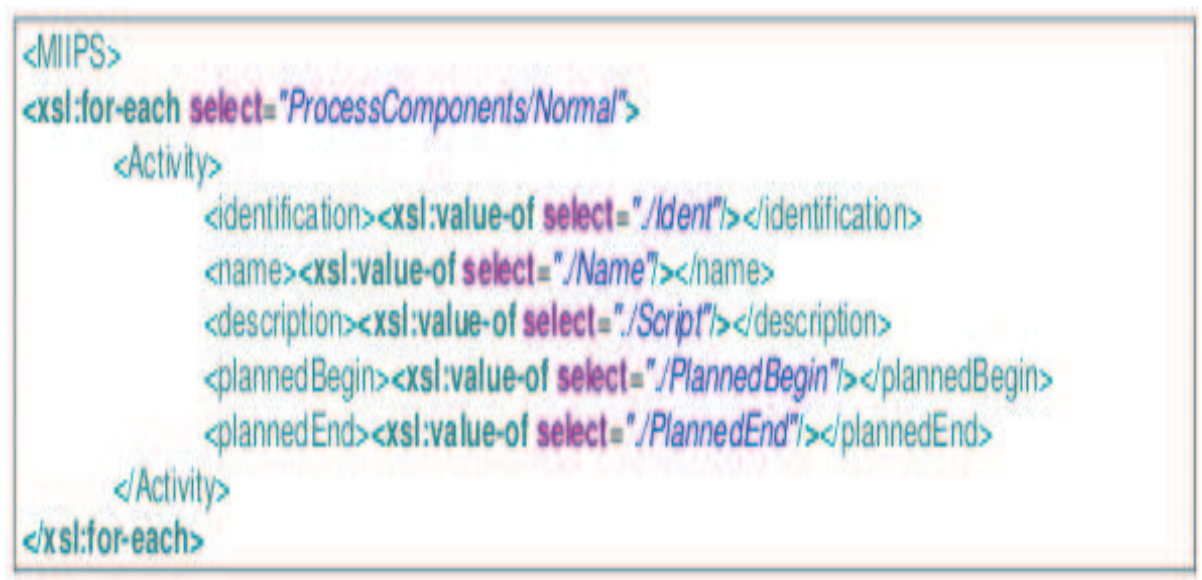

Figura 7. Trecho do template XSL da transformação WebAPSE em MIIPS

Na Figura 7 é ilustrado um trecho do template XSL responsável pela transformação de WebAPSEE para MIIPS. E Como já citado anteriormente, a ferramenta WebAPSEE não suportou o XML gerado para a mesma. Por isso, não foi possível ilustrar o documento XML gerado para o WebAPSEE importado na ferramenta em questão. Mas, as transformações entre os documentos foram realizadas.

\subsection{Parser de transformação}

O parser de transformação é o módulo responsável por ligar o modelo que se deseja transformar ao template XSL responsável pela transformação. Este módulo foi codificado utilizando a linguagem de programação Java.

O parser intermedia a transformação, basicamente, nele são indicados como valores de variáveis os caminhos dos diretórios onde se localiza o template XSL escolhido para a transformação, o modelo XML que será transformado e por último o diretório onde se deseja gravar o modelo XML resultado da transformação.

\section{Considerações Finais}

O objetivo geral deste trabalho, facilitar a troca de dados entre ferramentas de gerência de projetos através do modelo MIIPS, foi alcançado. Com o trabalho foi possível observar e sugerir melhorias ao modelo XML da ferramenta WebAPSEE, visando a ordenação de tarefas que pode ser algo a ser aplicado também em outras ferramentas. $\mathrm{O}$ MIIPS se demonstrou bem abrangente e útil ao engoblar os dados das duas ferramentas envolvidas.

Espera-se que as ferramentas MS Project e WebAPSEE possam ser vistas como exemplo para que outras ferramentas gestoras de projetos façam o uso do modelo MIIPS em seu desenvolvimento de software, e que as técnicas utilizadas nesse trabalham possam ser utilizadas em conjunto para a integração de ferramentas gestoras de projetos.

Este trabalho permite a realização de trabalhos futuros, citando:

- Para a integração de outras ferramentas, mapeamentos conceituais entre as ferramentas e o MIIPS e nvos templates XSL deverão ser desenvolvidos;

- Utilização de ontologias presente no trabalho de [Spínola 2004] para facilitar o 
mapeamento conceitual de esquemas XML.

- Possível alteração no modelo MIIPS para adequação a ferramenta MS Project;

- Possível alteração no XML do WebAPSEE em relação a ordenação de suas tarefas e atividades, ou o desenvolvimento de um parser de ordenação que trate as atividades paralelas;

- Possível correção no import XML do WebAPSEE;

- Desenvolvimento de interface gráfica para o parser de transformação para que facilite a utilização do usuário.

\section{Referências}

Carvalho, Daniel D. et al. (2011) "Apoio à Reutilização de Processos de Software em um Ambiente de Engenharia de Software Centrado em Processo", In X SIMPÓSIO BRASILEIRO DE QUALIDADE DE SOFTWARE. Curitiba, Paraná, p. 247-261.

Costa, A. et al. (2009) "Modelo Interoperável para Compartilhamento de Informações em Gerência de Projetos de Software", trabalho não publicado.

Definition of an Executable SPEM 2.0. Disponível em: $<$ http://ieeexplore.ieee.org/xpl/abstractReferences.jsp?

reload $=$ true $\& \mathrm{tp}=\&$ arnumber $=4425879 \&$ url $=\mathrm{http} \% 3 \mathrm{~A} \% 2 \mathrm{~F} \% 2 \mathrm{Fieeexplore.iee.}$. org \%2Fxpls\%2Fabs_all.jsp\%3Farnumber\%3D4425879> Acesso em: 25 de janeiro de 2014.

Kay, Michael (2002), XSLT: Referência do Programador, Alta Books, $2^{\mathrm{a}}$ edição.

López, Oscar C. (2008). Introdução ao Microsoft Project. In Universidade do Sul de Santa Catarina. Florianópolis, Santa Catarina.

Nascimento, Alexandre Erasmo K. (2001) "Intercâmbio de Dados entre Aplicativos Utilizando XML/XSLT", Tese de Mestrado em Informática, Universidade Federal do Rio Grande do Sul, Instituto de Informática, Porto Alegre.

Project Management Institute. (2010) "O Estudo de Benchmarking em Gerenciamento de Projetos Brasil".

Spinóla, Rodrigo O. (2004) Uma Infra-Estrutura para Integração de Ferramentas CASE baseada em XML, esquemas e ontologias", Tese de Mestrado em Ciências em Engenharia de Sistemas de Computação, Universidade Federal do Rio de Janeiro, Rio de Janeiro.

Yang, H. (2005), Advances in UML and XML-based Software Evolution, Idea Group Inc, $1^{\text {a }}$ edição. 